\title{
THE CLASSIFICATION OF TWO-DIMENSIONAL MANIFOLDS \\ BY
}

\author{
EDWARD M. BROWN AND ROBERT MESSER
}

\begin{abstract}
Invariants are constructed to classify all noncompact 2-manifolds including those with boundary. The invariants of a 2-manifold $M$ are the space of ends of $M$ and the subspaces of nonplanar ends, of nonorientable ends, and of ends that are limits of compact boundary components. Also the space of ends of the boundary components together with its natural map into the ends of $M$ and the orientation of these ends induced by orientations of neighborhoods of the orientable ends of $M$ are used in addition to the usual compact invariants. Special properties are established for the invariants of a 2-manifold, and a 2-manifold is constructed for each set of invariants with the special properties.
\end{abstract}

In 1963 Ian Richards gave a proof [5] of a classification theorem for open 2-manifolds. This proof used the compactness of the space of ends of a manifold to overcome the difficulties in the original proof [4] given by Kerékjártó in 1923. In 1971 a paper [3] by Martin Goldman gave algebraic criteria that also classified open 2-manifolds. Neither of these papers considered the complications that arise when the boundary of the 2-manifold is nonempty. In 1974 Margarida Barros [1] extended the classification theorem to 2-manifolds with compact boundary components. This result is also proved in the appendix to a paper [6] by Richard Tondra.

For an open 2-manifold the primary geometric invariants are the space of ends, the subset of nonplanar ends, and the subset of nonorientable ends, as defined in $\$ 1$. These invariants are far from sufficient to distinguish between the 2-manifolds that result from the various ways of removing a convergent sequence of points and the limit point from the boundary of a disk.

Additional complications arise when we consider 2-manifolds with nonplanar ends. A 2-manifold can be modified by removing a sequence of open disks converging to certain ends and then using tubes to connect various sequences of pairs of the resulting holes. The end space of the resulting 2-manifold is a quotient space of the end space of the original 2-manifold. In particular it is easy to create 2-manifolds with ends that have several, or even

Presented to the Society, January 5, 1978; received by the editors January 12, 1978 and, in revised form, December 20, 1978.

AMS (MOS) subject classifications (1970). Primary 57A05, 57C25; Secondary 05C10, 55A15, $55 \mathrm{~A} 20$. 
countably many, noncompact boundary components converging to them.

Such examples show that we need invariants that will reflect the way in which ends of noncompact boundary components are related to the ends of the 2-manifold.

On the other hand suppose we start with two disks. From the boundary of one of them remove a point and a sequence of points that converges to the point. From the boundary of the other disk remove a Cantor set. Then modify both 2-manifolds by attaching tubes as described above so that each has only one end. Since each of the 2-manifolds now has a countable number of noncompact boundary components all leading to the single nonplanar end, it seems hopeless to find invariants that will distinguish them. Fortunately, these two 2-manifolds are homeomorphic.

Orientability and the possibility of a nonorientable 2-manifold having orientable ends present a different type of difficulty in the classification of 2-manifolds. For example, two distinct 2-manifolds can be constructed from an annulus by removing from each boundary component one point and a sequence of points converging monotonically to the point. In one case choose both sequences to converge clockwise; in the other case choose one sequence to converge clockwise and the other to converge counterclockwise. However, if we take the connected sum of such a 2-manifold with a projective plane, it is no longer possible to compare the directions in which the sequences converge and the two examples become homeomorphic. Instead of starting with an annulus, start with a plane with an infinite number of compact boundary components and delete from each boundary component a monotone sequence and its limit point. Then even after taking the connected sum with a projective plane, we can still distinguish the pattern of directions of convergence at the end corresponding to the end of the original plane. This pattern would only be destroyed if we used an infinite number of projective planes and made this end nonorientable.

A new kind of invariant is required to overcome such difficulties. For this purpose we will introduce a subset of the ends of the boundary components that indicates how orientations of neighborhoods of orientable ends of the 2-manifold agree with orientations of neighborhoods of ends of the boundary components.

$\$ 1$ describes the invariants of a 2-manifold and contains other definitions and preliminary results. This is followed in $\$ 2$ by the main lemma and the classification theorem. Notice that a 2 -manifold $M$ is contained in an open 2-manifold $M \cup(\partial M \times[0, \infty))$ as the complement of open annuli and open regular neighborhoods of disjoint proper rays. Thus any connected 2-manifold can be constructed by modifying a 2 -sphere similar to the way Richards describes in $\$ 5$ of [5] and then removing open regular neighborhoods of the 
appropriate proper rays. If a set of invariants of a 2-manifold $M$ is given, it is not difficult to determine the open 2-manifold corresponding to $M \cup(\partial M \times$ $[0, \infty))$. However, it is not clear how to choose the proper rays. The last three sections of this paper give a direct construction of a 2-manifold having a given set of invariants. $\$ 3$ develops some graph theory that is used in the construction. $\$ 4$ is concerned with conditions on the invariants that are satisfied by the invariants of a 2 -manifold. The construction is then given in the last section.

1. The diagram of a 2-manifold. A 2-manifold is a separable metric space such that each point has a neighborhood homeomorphic to a disk, that is, to a closed 2-cell. All manifolds and maps between manifolds are intended to be in the piecewise linear category. In this section we will use $M$ to denote an arbitrary connected 2-manifold. The boundary of $M$ will be denoted $\partial M$ and the union of the noncompact components of $\partial M$ will be denoted $d M$. A submanifold of $M$ is a subset of $M$ that is also a 2-manifold. For a submanifold $C$ of $M$ it is important to distinguish between the manifold boundary $\partial C$ and the topological frontier $\mathrm{fr} C$, and between the manifold interior Int $C$ and the topological interior int $C$.

Let $\underline{\pi}_{0}(X)$ denote the space of ends of $X$. Suppose $Q$ is a complementary domain of a compact submanifold $C$ of a 2-manifold $M$. That is, $Q$ is the closure of one of the components of $M-C$. Then $Q^{*}$ will denote the subset of ends in $\pi_{0}(M)$ that correspond to ends of $Q$. Since $Q$ is a closed subset of $M$, the inclusion $i: Q \rightarrow M$ is a proper map and $Q^{*}=i_{\#}\left(\pi_{0}(Q)\right)$. Notice that if $Q$ is compact, then $Q^{*}=\varnothing$. Recall that a basis for the topology of $\pi_{0}(M)$ is formed by the sets $Q^{*}$ where $Q$ is a complementary domain of a compact submanifold of $M$. An end in $\pi_{0}(M)$ is planar if and only if it is in $Q^{*}$ where $Q$ is planar. Similarly an end in $\pi_{0}(M)$ is orientable if and only if it is in $Q^{*}$ where $Q$ is orientable. Let $\mathcal{H}$ denote the set of nonplanar ends of $M$, let $\mathscr{K}$ denote the set of nonorientable ends of $M$, and let $\mathcal{S}$ denote the set of ends of $M$ that are limits of compact components of $\partial M$. Notice that $\mathscr{K} \subseteq \mathcal{H}$ and that $\mathcal{K}, \mathcal{K}$, and $\mathcal{S}$ are closed subsets of $\underline{\pi}_{0}(M)$. For further information see $\$ 1.2$ of the paper [2] by Brown and Tucker and $\$ 3$ of the paper [5] by Richards.

If $p$ is a point of a component $b$ of $d M$, we will of ten identify the equivalence class $[p] \in \pi_{0}(d M)$ with the component $b$. Since each $b \in$ $\pi_{0}(d M)$ is homeomorphic to the real line, it has exactly two ends. Since $\pi_{0}(d M)$ is a discrete topological space, we can give $\pi_{0}(d M)$ the discrete topology and the function $e: \pi_{0}(d M) \rightarrow \pi_{0}(d M)$ that maps the end of any component $b$ of $d M$ to $b \in \pi_{0}(d M)$ will be continuous. We also define the map $v: \underline{\pi}_{0}(d M) \rightarrow \pi_{0}(M)$ induced by inclusion. An end of a component of $d M$ is mapped by $v$ to the corresponding end of $M$. 
An exhausting sequence for $M$ is a sequence $C_{0}, C_{1}, \ldots$ of compact submanifolds of $M$ such that

$$
\begin{gathered}
C_{0}=\varnothing, \\
C_{n} \subseteq \text { int } C_{n+1} \text { for } n=0,1, \ldots,
\end{gathered}
$$

and

$$
M=\bigcup_{n=0}^{\infty} C_{n} .
$$

Lemma 1.1. Suppose $V$ is an open subset of $\pi_{0}(M)$ and $\left\{V_{\alpha} \mid \alpha \in A\right\}$ is an open cover of $V$. Then there is a sequence $Q_{1}, Q_{2}, \ldots$ of disjoint complementary domains of compact submanifolds so that $\left\{Q_{1}^{*}, Q_{2}^{*}, \ldots\right\}$ refines $\left\{V_{\alpha} \mid \alpha \in \mathrm{A}\right\}$ and $Q_{1}^{*} \cup Q_{2}^{*} \cup \cdots=V$. Furthermore if each $V_{\alpha}$ is contained in the set of ends determined by a complementary domain $R_{\alpha}$ of some compact submanifold, then each $Q_{i}$ can be chosen so that it is contained in a complementary domain $R_{\alpha_{1}}$ of one of the compact submanifolds.

Proof. Let $C_{0}, C_{1}, \ldots$ be an exhausting sequence for $M$. We want to consider complementary domains of the submanifolds $C_{n}$ that determine subsets of the sets in the open cover $\left\{V_{\alpha} \mid \alpha \in \mathrm{A}\right\}$. Let $Q_{1}, Q_{2}, \ldots$ be the sequence that begins with all such complementary domains of $C_{0}$ and inductively lists all such complementary domains of $C_{n}$ that are not contained in any previously listed complementary domains. Then $\left\{Q_{1}^{*}, Q_{2}^{*}, \ldots\right\}$ refines $\left\{V_{\alpha} \mid \alpha \in \mathrm{A}\right\}$. Notice that a basis of $\pi_{0}(M)$ is determined by the collection of all complementary domains of the $C_{n}$ and that the partition of $\underline{\pi}_{0}(M)$ determined by the complementary domain of $C_{n+1}$ refines the partition determined by the complementary domains of $C_{n}$. It follows from these two facts that $Q_{1}^{*} \cup Q_{2}^{*} \cup \cdots=V$.

Now suppose that $Q_{i}^{*} \subseteq V_{\alpha_{i}} \subseteq R_{\alpha_{i}}^{*}$ where $R_{\alpha_{i}}$ is a complementary domain of some compact submanifold. Consider the noncompact complementary domains of a regular neighborhood of the union of the compact submanifolds that determine $Q_{i}$ and $R_{\alpha_{i}}$. Those that are contained in $Q_{i}$ must also be contained in $R_{\alpha_{i}}$ and they determine a partition of $Q_{i}^{*}$. Replacing each $Q_{i}$ with such a collection of complementary domains produces a sequence that also satisfies the final conclusion of the lemma.

An orientation of $\underline{\pi}_{0}(d M)$ is a subset $\mathscr{P} \subseteq \underline{\pi}_{0}(d M)$ such that if $x \in \pi_{0}(M)$ - $K$, then for some orientable complementary domain $Q$ of a compact submanifold with $x \in Q^{*}$, an orientation of $Q$ agrees with $\mathscr{P}$. That is, a proper ray in $d M \cap Q$ determines an element of $\mathscr{P}$ if and only if the direction of the ray is consistent with the orientation induced from the orientation on $Q$. If $M$ is orientable, we require an orientation of $M$ to agree with $\mathscr{P}$. 
It follows from Lemma 1.1 that there is a sequence $Q_{1}, Q_{2}, \ldots$ of disjoint orientable complementary domains of compact submanifolds of $M$ so that $Q_{1}^{*} \cup Q_{2}^{*} \cup \cdots=\pi_{0}(M)-\mathscr{K}$. If $M$ is orientable, then $\mathscr{K}=\varnothing$ and we can choose the sequence to consist of one set $Q_{1}$ with $Q_{1}^{*}=\pi_{0}(M)$. A choice of orientation on each $Q_{i}$ will then define an orientation $\mathscr{\rho}$ of $\pi_{0}(d M)$ that agrees with these orientations. On the other hand suppose $\mathcal{P}$ and $\mathcal{P}^{\prime}$ are two orientations of $\underline{\pi}_{0}(d M)$. Then for each $x \in \underline{\pi}_{0}(M)-\mathcal{K}$, there is an orientable complementary domain $Q$ of a compact submanifold such that $x \in Q^{*}$ and $Q$ has an orientation that agrees with $\mathcal{P}$ and an orientation that agrees with $\mathscr{P}^{\prime}$. Thus we can use Lemma 1.1 to obtain a sequence $Q_{1}, Q_{2}, \ldots$ of disjoint orientable complementary domains of compact submanifolds of $M$ so that $Q_{1}^{*} \cup Q_{2}^{*} \cup \cdots=\pi_{0}(M)-\mathcal{K}$, and each $Q_{i}$ has an orientation that agrees with $\mathscr{P}$ and an orientation that agrees with $\mathscr{P}^{\prime}$.

The diagram of $M$ consists of the following spaces and maps. The unlabeled maps are inclusions and $\mathscr{P}$ is an orientation of $\underline{\pi}_{0}(d M)$.

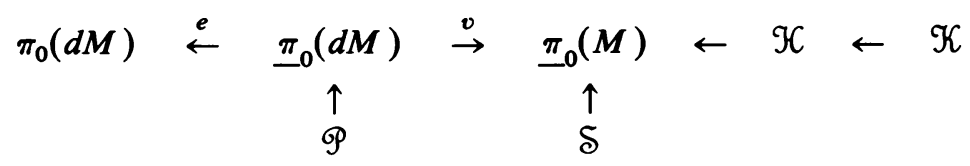

The diagram of $M$ will be denoted $\Delta M$. It contains the primary invariants for a 2-manifold. The only other information needed to determine the homeomorphism type of $M$ will be the genus, orientability type, and number of compact boundary components. The genus will be finite if and only if $\mathcal{H}=\varnothing$. There are four orientability types: orientable, finitely nonorientable of even type, finitely nonorientable of odd type, and infinitely nonorientable. One of the first three types will occur if and only if $\mathcal{K}=\varnothing$. The number of compact boundary components will be finite if and only if $\delta=\varnothing$. Additional information can be found in $\$ \S 2$ and 3 of the paper [5] by Richards.

LEMMA 1.2. Suppose $V$ is an open and closed subset of $\pi_{0}(M)$ and $\left\{V_{\alpha} \mid \alpha \in\right.$ A) is an open cover of $V$. Then there is a compact submanifold $D$ of $M$ with complementary domains $R_{1}, \ldots, R_{n}$ so that for some $m<n,\left\{R_{1}^{*}, \ldots, R_{m}^{*}\right\}$ refines $\left\{V_{\alpha} \mid \alpha \in \mathrm{A}\right\}$ and $R_{1}^{*} \cup \cdots \cup R_{m}^{*}=V$. Furthermore if each $V_{\alpha}$ is contained in the set of ends determined by a complementary domain of some compact submanifold, then $D$ can be chosen so that each of $R_{1}, \ldots, R_{n}$ is contained in a complementary domain of one of the compact submanifolds.

Proof. Since $V$ is compact, the sequence $Q_{1}, Q_{2}, \ldots$ of disjoint complementary domains of compact submanifolds obtained from Lemma 1.1 will be a finite sequence. Let $D$ be any compact submanifold of $M$ that contains the finite number of compact submanifolds involved in defining $Q_{1}, Q_{2}, \ldots$ Let $R_{1}, \ldots, R_{m}$ be the complementary domains of $D$ that are contained in any 
one of the sets $Q_{1}, Q_{2}, \ldots$ Then $\left\{R_{1}^{*}, \ldots, R_{m}^{*}\right\}$ refines $\left\{Q_{1}^{*}, Q_{2}^{*}, \ldots\right\}$ so it refines $\left\{V_{\alpha} \mid \alpha \in \mathrm{A}\right\}$. Also $R_{1}^{*} \cup \cdots \cup R_{m}^{*}=Q_{1}^{*} \cup Q_{2}^{*} \cup \cdots=V$.

Notice that any compact submanifold containing $D$ will also satisfy the conclusion of this lemma.

Suppose $\mathcal{P}$ is an orientation of $\pi_{0}(d M)$. We will be concerned with the following properties of a submanifold $C$ of $M$ with complementary domains $Q_{i}, i=1, \ldots, m$. The number of elements in a set $X$ will be denoted by $\#$.

(1) a. The submanifold $C$ is compact and connected.

b. If $b \in \pi_{0}(\partial M)$, then no component of $\mathrm{cl}(b-C)$ is an arc.

c. If $Q_{i} \cap d M \neq \varnothing$, then the components of fr $Q_{i}$ are arcs.

(2) a. Each $Q_{i}$ is noncompact.

b. Each $Q_{i}$ is either planar or of infinite genus.

c. Each $Q_{i}$ is either orientable or infinitely nonorientable.

d. Each $Q_{i}$ contains either no compact components of $\partial M$ or an infinite number.

(3) If $Q_{i}$ is orientable, then an orientation of $Q_{i}$ agrees with $\mathscr{P}$.

(4) If $C \subseteq D$ for some compact submanifold $D$, then

$$
\# \pi_{0}(\operatorname{fr} C)-\# \pi_{0}(M-C)<\# \pi_{0}(\text { fr } D)-\# \pi_{0}(M-D) \text {. }
$$

LEMMA 1.3. Let $B$ be a compact submanifold of $M$. There is a submanifold $C$ of $M$ such that $B \subseteq$ int $C$ and $C$ satisfies properties (1), (2), (3) and (4).

Proof. Notice for any nonempty compact submanifold $C$ of $M$ that $\# \pi_{0}(\mathrm{fr} C)-\# \pi_{0}(M-C) \geqslant 0$. Begin by letting $C$ be a compact submanifold such that $B \subseteq$ int $C$ and $C$ satisfies property (4). We will proceed to modify $C$ so that it also satisfies properties (3), (1), and (2).

Suppose $Q_{i}$ is an orientable or finitely nonorientable complementary domain of $C$. For each $x \in Q_{i}^{*}$ there is an orientable complementary domain $Q$ of a compact submanifold so that $x \in Q^{*}$ and an orientation of $Q$ agrees with $\mathscr{P}$. We can assume $Q \subseteq Q_{i}$. Thus by Lemma 1.2 we can find a compact submanifold $D$ so that $C \subseteq$ int $D$ and if $R_{1}, \ldots, R_{m}$ are the complementary domains of $D$ that are contained in $Q_{i}$, then $R_{1}, \ldots, R_{m}$ are orientable and have orientations that agree with $\mathscr{P}$. We can also choose $D$ so that the number \# $\pi_{0}\left(\mathrm{fr}\left(R_{1} \cup \cdots \cup R_{m}\right)\right)-m$ cannot be decreased by enlarging $D$. Adjoin $D \cap Q_{i}$ to $C$.

After the operation described in the previous paragraph has been performed for each of the orientable and finitely nonorientable complementary domains of $C$, property (3) will be satisfied. These operations may increase the difference between the number of frontier components and the number of complementary domains. Nevertheless property (4) will still be satisfied since for each complementary domain the corresponding difference cannot be decreased by enlarging $C$. 
The modification of $C$ so that it satisfies property (1) will involve adjoining to $C$ a regular neighborhood of an arc in a complementary domain of $C$. The arc will be a spanning arc of the complementary domain and have endpoints on different components of $\operatorname{fr} C$, or it will be an arc in $\partial M$, or it will be an arc that spans from a simple closed curve component of fr $Q_{i}$ to a component of $d M$. This modification will not increase \# $\pi_{0}(\mathrm{fr} C)$ or decrease $\# \pi_{0}(M-C)$; so the resulting submanifold will still satisfy property (4). Since $C$ now satisfies property (4), it follows that $\# \pi_{0}(\mathrm{fr} C)$ and $\# \pi_{0}(M-C)$ must remain constant when such strips are attached. In particular no new orientable complementary domains will be created. Thus the resulting submanifold will still satisfy property (3).

Now adjoin strips between appropriate components of fr $C$ so that $C$ will be connected. If $b \in \pi_{0}(\partial M)$ and $b \cap C \neq \varnothing$, adjoin strips to $C$ along any components of $\operatorname{cl}(b-C)$ that are arcs. Suppose $Q_{i} \cap d M \neq \varnothing$. We can assume there is a component of fr $Q_{i}$ that is an arc. Indeed if there are none, adjoin to $C$ a strip in $Q_{i}$ from one of the simple closed curve components of fr $Q_{i}$ to a component of $d M$. Now it would be possible to adjoin to $C$ disjoint strips between such an arc and any simple closed curve components of fr $Q_{i}$. The submanifold $C$ thus satisfies properties (1), (3), and (4).

To attain property (2) first adjoin to $C$ any compact complementary domains. Because the operation described in the second paragraph of this proof was applied to any finitely nonorientable complementary domains, the submanifold $C$ now satisfies property (2c). For any complementary domain $Q_{i}$ of finite genus, choose a maximal collection of disjoint pairs of transverse simple closed curves in $Q_{i}$. Use strips to adjoin to $C$ regular neighborhoods of the pairs of simple closed curves in this finite collection. For any complementary domain $Q_{i}$ that contains only a finite number of compact components of $\partial M$, use strips to adjoin to $C$ regular neighborhoods of any compact component of $Q_{i} \cap \partial M$. After these modifications, $C$ will satisfy property (2) as well as properties (1), (3), and (4).

2. The classification theorem. The main lemma, stated below, will be used inductively in the proof of the classification theorem for 2-manifolds. In this section we will often refer to the four properties stated in the previous section. The remarks following the statement of Lemma 2.1 describe the method of combining an isomorphism between 2-manifold diagrams with a homeomorphism between submanifolds to produce isomorphisms between the diagrams of the complementary domains of the submanifolds.

LEMMA 2.1. Suppose $M$ and $M^{\prime}$ are connected 2-manifolds that have the same genus, orientability type, and number of compact boundary components. Suppose $h: \Delta M \rightarrow \Delta M^{\prime}$ is an isomorphism of diagrams. Suppose $f:(C$, fr $C) \rightarrow$ $\left(C^{\prime}\right.$, fr $\left.C^{\prime}\right)$ is a homeomorphism where either $C=C^{\prime}=\varnothing$ or $C \subseteq M$ and 
$C^{\prime} \subseteq M^{\prime}$ are submanifolds that satisfy properties (1), (2) and (3). Suppose that $Q_{1}, \ldots, Q_{m}$ are the complementary domains of $C$, that $Q_{1}^{\prime}, \ldots, Q_{m}^{\prime}$ are the complementary domains of $C^{\prime}$, and for $i=1, \ldots, m$, that $h$ and $f$ induce isomorphisms $h_{i}: \Delta Q_{i} \rightarrow \Delta Q_{i}^{\prime}$. Suppose $D^{\prime}$ is a submanifold of $M^{\prime}$ with complementary domains $R_{1}^{\prime}, \ldots, R_{n}^{\prime}$ such that

$C^{\prime} \subseteq$ int $D^{\prime}$,

$D^{\prime}$ satisfies properties (1), (2), (3) and (4), and

$Q_{i}^{\prime} \cap D^{\prime}$ is connected for $i=1, \ldots, m$.

Then there is a submanifold $D$ of $M$ such that

$C \subseteq$ int $D$,

$D$ satisfies properties (1), (2) and (3),

$f$ extends to a homeomorphism $\mathrm{g}:(D, \mathrm{fr} D) \rightarrow\left(D^{\prime}, \mathrm{fr} D^{\prime}\right)$, and the complementary domains of $D$ can be labeled $R_{1}, \ldots, R_{n}$ so that, for $j=1, \ldots, n$, $h$ and $g$ induce isomorphisms $\Delta R_{j} \rightarrow \Delta R_{j}^{\prime}$.

The isomorphisms $h_{i}: \Delta Q_{i} \rightarrow \Delta Q_{i}^{\prime}$ are defined as follows. Consider a component $b$ of $d Q_{i}$ that contains an arc $A$ of $\operatorname{fr} Q_{i}$. Let $p$ and $q$ be the endpoints of $A$. Then $p$ and $q$ are the initial points of proper rays of $d M \cap Q_{i}$. Let $p^{*}$ and $q^{*}$ denote the ends in $\pi_{0}(d M)$ determined by the respective rays. We require that $h[p]=[f(p)]$ and $h[q]=[f(q)]$ in $\pi_{0}\left(d M^{\prime}\right)$, and that $h\left(p^{*}\right)=f(p)^{*}$ and $h\left(q^{*}\right)=f(q)^{*}$ in $\pi_{0}\left(d M^{\prime}\right)$. Then we define $h_{i}(b)$ to be the component of $d Q_{i}^{\prime}$ that contains $f(A)$. Elements of $\pi_{0}\left(d Q_{i}\right)$ and $\pi_{0}\left(d Q_{i}^{\prime}\right)$ that do not contain frontier arcs will be identified with the corresponding elements of $\pi_{0}(d M)$ and $\pi_{0}\left(d M^{\prime}\right)$. Similarly ends in $\underline{\pi}_{0}\left(Q_{i}\right), \underline{\pi}_{0}\left(Q_{i}^{\prime}\right), \underline{\pi}_{0}\left(d Q_{i}\right)$, and $\underline{\pi}_{0}\left(d Q_{i}^{\prime}\right)$ will be identified with the corresponding ends in $\underline{\pi}_{0}(M), \underline{\pi}_{0}\left(M^{\prime}\right)$, $\underline{\pi}_{0}(d \bar{M})$, and $\underline{\pi}_{0}\left(d M^{\prime}\right)$. On these sets we define $h_{i}$ to be the restriction of $h$. The resulting map $h_{i}: \Delta Q_{i} \rightarrow \Delta Q_{i}^{\prime}$ will then be an isomorphism. Similar requirements and conventions apply to the definition of the isomorphisms $\Delta R_{j} \rightarrow \Delta R_{j}^{\prime}$ induced by $h$ and $g$.

Proof of Lemma 2.1. Begin by using Lemmas 1.2 and 1.3 to construct a compact submanifold $D$ of $M$ so that

$C \subseteq$ int $D$,

$D \cap Q_{i}$ is connected,

$D \cap Q_{i}$ is nonorientable if $Q_{i}$ is nonorientable,

$D$ intersects $b \in \pi_{0}(d M)$ whenever $D^{\prime}$ intersects $h(b) \in \pi_{0}\left(d M^{\prime}\right)$,

the complementary domains of $D$ can be labeled $R_{j 1}, \ldots, R_{j m}$ for $j=$ $1, \ldots, n$ so that $R_{j 1}^{*} \cup \cdots \cup R_{j m}^{*}=h^{-1}\left(R_{j}^{\prime *}\right)$, and

$D$ satisfies properties (1), (2), (3) and (4).

Let $R_{j}=R_{j 1} \cup \cdots \cup R_{j m}$. Notice for each $j=1, \ldots, n$, that $R_{j} \subseteq Q_{i}$ for some $i$. This is because

for some $i$.

$$
R_{j}^{*}=h^{-1}\left(R_{j}^{\prime *}\right) \subseteq h^{-1}\left(Q_{i}^{\prime *}\right)=Q_{i}^{*}
$$


The first modifications of $D$ will have the effect of joining together the components of $R_{j}$ so that the frontier components will correspond to the frontier components of $R_{j}^{\prime}$. There are two cases to consider depending on whether or not $R_{j}^{\prime} \cap d M^{\prime}=\varnothing$.

Suppose $R_{j}^{\prime} \cap d M^{\prime}=\varnothing$. Then fr $R_{j}^{\prime}$ consists of simple closed curves; by property (4) there is only one. Since $R_{j}^{\prime *}$ contains no ends corresponding to ends of $d M$, neither does $R_{j}^{*}$. Therefore by property (1b) $R_{j} \cap d M=\varnothing$; so fr $R_{j}$ consists of simple closed curves. Find disjoint strips in $D \cap Q_{i}$ that join one of these simple closed curves to each of the others. If $Q_{i}$ is nonorientable, notice that since $D$ was chosen so that $D \cap Q_{i}$ is also nonorientable, these strips can be chosen so any orientations on the complementary domains $R_{j 1}, \ldots, R_{j m}$, will match when the complementary domains are joined by these strips. After these strips are adjoined to $R_{j}$, the resulting submanifold $D$ will still satisfy the properties mentioned at the beginning of the proof. In addition the resulting $R_{j}$ will be connected, fr $R_{j}$ will be a single simple closed curve, and $R_{j}^{*}=h^{-1}\left(R_{j}^{\prime *}\right)$.

Suppose $R_{j}^{\prime} \cap d M^{\prime} \neq \varnothing$. Then the components of fr $R_{j}^{\prime}$ are arcs $A_{1}^{\prime}, \ldots, A_{r}^{\prime}$. Let $p_{k}^{\prime}$ and $q_{k}^{\prime}$ denote the endpoints of $A_{k}^{\prime}$. It follows from our choice of $D$ that the components of fr $R_{j}$ are arcs, and that we can label $2 r$ of the endpoints of these arcs $p_{k}$ and $q_{k}, k=1, \ldots, r$, so that $h\left(p_{k}^{*}\right)=p_{k}^{\prime *}$ and $h\left(q_{k}^{*}\right)=q_{k}^{*}$. Let $p$ be any other endpoint of an arc of fr $R_{j}$. Then $p^{*}$ corresponds to an end in $R_{j}^{*}$. So one of the ends of $h[p] \in \pi_{0}\left(d M^{\prime}\right)$ corresponds to an end in $R_{j}^{\prime *}$. But since $D^{\prime} \cap h[p]=\varnothing$, both ends of $h[p]$ must correspond to ends in $R_{j}^{\prime *}$. So both ends of $[p]$ correspond to ends in $R_{j}^{*}$. Therefore we can modify $D$ by removing an open regular neighborhood in $D-C$ of $D \cap[p]$. This process can be repeated until all but $r$ arcs of fr $R_{j}$ remain. These arcs must join pairs of the points $p_{k}, q_{k}, k=1, \ldots, r$.

The above process may create frontier components that are simple closed curves. Remove from $D$ an open strip in $D-C$ that joins each such frontier component to a frontier component that is an arc. If $Q_{i}$ is nonorientable, notice again that these strips can be chosen so any orientations on the complementary domains $R_{j 1}, \ldots, R_{j m}$, will match when the complementary domains are joined by these strips.

These modifications of $D$ to join together various of the original complementary domains $R_{j 1}, \ldots, R_{j m}$ are to be performed for each $j=1, \ldots, n$. The following argument shows that at this stage in the construction, $m_{j}=1$ for each $j=1, \ldots, n$. That is, the original $R_{j 1}, \ldots, R_{j m}$ will have been joined together to form a single complementary domain $R_{j 1}=R_{j}$ of the resulting submanifold $D$.

First we can find a compact submanifold of $M^{\prime}$ containing $D^{\prime}$ and having complementary domains that determine a refinement of $\left\{h\left(R_{j k}^{*}\right) \mid j=\right.$ 
$\left.1, \ldots, n ; k=1, \ldots, m_{j}\right\}$. By adding strips, we can also assume that any simple closed curve frontier component of this compact submanifold is the entire frontier of the complementary domain it bounds. As remarked in the second paragraph of this proof, the complementary domains of this compact submanifold whose ends make up the image of the ends of a complementary domain of $D$ will all be contained in a single complementary domain of $D^{\prime}$. Thus in $M^{\prime}-D^{\prime}$ we can apply the process of eliminating arc frontier components from the compact submanifold until it has the same number of arc frontier components as $D$. Now its complementary domains still determine a refinement of $\left\{h\left(R_{j k}^{*}\right) \mid j=1, \ldots, n ; k=1, \ldots, m_{j}\right\}$. So the number of complementary domains with arc frontier components determined by the compact submanifold in $M^{\prime}$ is greater than or equal to the number determined by $D$ in $M$, and this is greater than or equal to the number determined by $D^{\prime}$ in $M^{\prime}$. Yet for all three submanifolds there is a one-to-one correspondence between simple closed curve frontier components and complementary domains that have simple closed curve frontier components. Also, all three submanifolds have the same number of arc frontier components. Because $D^{\prime}$ satisfies property (4), it follows that the three submanifolds must have the same number of complementary domains with arc frontier components. Therefore the complementary domains of $D$ must be in one-to-one correspondence with the complementary domains of $D^{\prime}$.

Suppose now that $R_{j}$ is orientable. We want to show that $R_{j}$ has an orientation that agrees with $\mathscr{P}$. If $Q_{i}$ is orientable, then an orientation on it agrees with $\mathscr{P}$. This is true even if $C=\varnothing$, since then $M$ is orientable and we required in this case that an orientation of $M$ must agree with $\mathscr{P}$. Since $R_{j}$ is contained in $Q_{i}$, the orientation induced on $R_{j}$ will also agree with $\mathscr{P}$. Suppose $Q_{i}$ is nonorientable. Since all the ends in $R_{j}^{*}$ are orientable, all the ends in $h\left(R_{j}^{*}\right)=R_{j}^{\prime *}$ are also orientable. Thus $R_{j}^{\prime}$ is orientable and has an orientation that agrees with $h(\mathscr{P})$. If an open regular neighborhood of an arc of a boundary component $b \in \pi_{0}(d M)$ was removed from $D$ to join together $R_{j k}$ and $R_{j l}$, then exactly one end of $b$ is in $\mathscr{P}$ since exactly one end of $h(b)$ is in $h(\mathscr{P})$. Since $R_{j k}$ and $R_{j l}$ have orientations that agree with $\mathscr{P}$, these orientations are compatible when these complementary domains are joined by the strip. If an open strip was removed from $D$ to join together $R_{j k}$ and $R_{j l}$, then as noted previously we can assume the strip was chosen so that the orientations of $R_{j k}$ and $R_{j l}$ are compatible. In any case the orientation of the resulting complementary domain agrees with $\mathscr{P}$.

Returning to the case that $R_{j}^{\prime} \cap d M^{\prime} \neq \varnothing$, we have that fr $R_{j}^{\prime}$ consists of arcs $A_{1}^{\prime}, \ldots, A_{r}^{\prime}$ with $\partial A_{k}^{\prime}=\left\{p_{k}^{\prime}, q_{k}^{\prime}\right\}$ for $k=1, \ldots, r$. Now fr $R_{j}$ also consists of arcs $A_{1}, \ldots, A_{r}$ such that $\partial A_{1} \cup \cdots \cup \partial A_{r}=\left\{p_{1}, q_{1}, \ldots, p_{r}, q_{r}\right\}$ with $h\left(p_{k}^{*}\right)=p_{k}^{\prime *} \in \underline{\pi}_{0}\left(d M^{\prime}\right)$ and $h\left(q_{k}^{*}\right)=q_{k}^{\prime *} \in \underline{\pi}_{0}\left(d M^{\prime}\right)$. We want to modify 
the $\operatorname{arcs} A_{k}$ so that $\partial A_{k}=\left\{p_{k}, q_{k}\right\}$ for $k=1, \ldots, r$.

For a fixed value of $k$ we can assume the components of $\mathrm{fr} \boldsymbol{R}_{j}$ are indexed so that $p_{k} \in \partial A_{k}$. But we might not have $q_{k} \in \partial A_{k}$. To remedy this choose a strip in $R_{j}$ between $A_{k}$ and the component of fr $R_{j}$ that contains $q_{k}$. Notice that such a strip cannot separate $R_{j}$. Indeed, as before, we can use the fact that $D^{\prime}$ satisfies property (4) to show that when this strip is added to $D$, the number of complementary domains equals the number of complementary domains of $D^{\prime}$ in $M^{\prime}$. If $R_{j}$ is nonorientable, we can choose the strip so that one of the resulting frontier components is an arc from $p_{k}$ to $q_{k}$. If $R_{j}$ is orientable, then $R_{j}^{\prime}$ is orientable; so exactly one of $p_{k}^{\prime *}$ and $q_{k}^{\prime *}$ is in $h(\mathscr{P})$. Thus exactly one of $p_{k}^{*}$ and $q_{k}^{*}$ is in $\mathscr{P}$. Since we know $R_{j}$ has an orientation that agrees with $\mathscr{P}$, it follows that when the strip is added to $D$, one of the resulting frontier components will be an arc from $p_{k}$ to $q_{k}$. This process can be repeated for each of the components of fr $R_{j}$ that does not join $p_{k}$ and the corresponding $q_{k}$.

We now have that the restrictions of $h$ and the correspondence between fr $R_{j}$ and fr $R_{j}^{\prime}$ give isomorphisms $\Delta R_{j} \rightarrow \Delta R_{j}^{\prime}$ for $j=1, \ldots, n$. We want to make a final modification to $D$ so that for each $i=1, \ldots, m$, there will be a homeomorphism of $D \cap Q_{i}$ onto $D^{\prime} \cap Q_{i}^{\prime}$ that extends $f \mid$ fr $C$ and the correspondences between fr $R_{j}$ and fr $R_{j}^{\prime}$ for $j=1, \ldots, n$.

In the case that $C$ and $C^{\prime}$ are nonempty, if $D \cap Q_{i}$ or $D^{\prime} \cap Q_{i}^{\prime}$ is nonorientable, nonplanar, or contains compact boundary components of $M$ or $M^{\prime}$, then $Q_{i}^{*}$ or $Q_{i}^{\prime *}$ has an end that is nonorientable, nonplanar, or is a limit of compact boundary components, respectively. Since $Q_{i}^{*}$ and $Q_{i}^{\prime *}$ contain the same types of ends, we can adjust the orientability type, genus, and number of compact components of $\partial M$ in $D \cap Q_{i}$ as follows. Choose a regular neighborhood of appropriate one-sided simple closed curves, pairs of transverse simple closed curves, and compact components of $\partial M$ in $D \cap Q_{i}$ or $Q_{i}-D$. Then for each component of the regular neighborhood, either remove from $D \cap Q_{i}$ the interior of the component and an open strip joining it to fr $R_{j}$, or add to $D$ the component and a strip joining it to fr $R_{j}$.

In the case that $C=C^{\prime}=\varnothing$, if all the ends of $M$ are planar, then $M$ will have finite genus and by hypothesis $M^{\prime}$ will have the same finite genus. Thus $D$ and $D^{\prime}$ have the same genus. Similarly if all the ends of $M$ are orientable, then $D$ and $D^{\prime}$ will have the same orientability type; and if none of the ends of $M$ are limits of compact components of $\partial M$, then $D$ and $D^{\prime}$ will have the same number of compact components of $\partial M$. Otherwise we can adjust $D$ as described in the previous paragraph.

Now a component of $\partial\left(D \cap Q_{i}\right)$ is either a simple closed curve component of $\partial M$ or of $\operatorname{fr} C \cup$ fr $D$, or it is made up of arcs of $d M$ alternating with arcs of fr $C \cup$ fr $D$. A component of $\partial\left(D^{\prime} \cap Q_{i}^{\prime}\right)$ similarly is either a simple 
closed curve component of $\partial M^{\prime}$ or of fr $C^{\prime} \cup$ fr $D^{\prime}$, or it is made up of arcs of $d M^{\prime}$ alternating with arcs of fr $C^{\prime} \cup$ fr $D^{\prime}$. The endpoints of arcs of fr $C \cup$ fr $D$ determine ends in $\pi_{0}(d M)$. The corresponding endpoints of arcs of fr $C^{\prime} \cup$ fr $D^{\prime}$ determine corresponding ends in $\pi_{0}\left(d M^{\prime}\right)$. Thus we can extend $f: C \rightarrow C^{\prime}$ to a homeomorphism between $C \cup \bar{\cup} D$ and $C^{\prime} \cup \partial D^{\prime}$ that agrees with the correspondence between fr $C \cup$ fr $D$ and fr $C^{\prime} \cup$ fr $D^{\prime}$ and the correspondence between arcs of $d M$ and arcs of $d M^{\prime}$. Since $D \cap Q_{i}$ and $D^{\prime} \cap Q_{i}^{\prime}$ have the same genus, orientability type, and number of compact components of $\partial M$ or $\partial M^{\prime}$, we can further extend $f$ to a homeomorphism $g$ : $D \rightarrow D^{\prime}$.

THEOREM 2.2. Two connected 2-manifolds are homeomorphic if and only if they have the same genus, the same orientability type, the same number of compact boundary components, and isomorphic diagrams.

Proof. Let $M$ and $M^{\prime}$ denote the two 2-manifolds. Suppose there is a homeomorphism between $M$ and $M^{\prime}$. Then $M$ and $M^{\prime}$ have the same genus, orientability type, and number of compact boundary components. There is a sequence of disjoint oriented complementary domains of compact submanifolds of $M$ that determine an orientation of $\underline{\pi}_{0}(d M)$. The image under the homeomorphism of these oriented complementary domains will determine an orientation of $\underline{\pi}_{0}\left(d M^{\prime}\right)$. With these orientations on $\underline{\pi}_{0}(d M)$ and $\underline{\pi}_{0}\left(d M^{\prime}\right)$, the homeomorphism will induce an isomorphism between the diagrams of $M$ and $M^{\prime}$.

To prove the converse, let $h: \Delta M \rightarrow \Delta M^{\prime}$ be an isomorphism of diagrams. Let $B_{k}, k=0,1, \ldots$, be an exhausting sequence for $M$ and let $B_{k}^{\prime}, k=$ $0,1, \ldots$, be an exhausting sequence for $M^{\prime}$. Let $C_{0}=C_{0}^{\prime}=\varnothing$ and let $f_{0}$ : $C_{0} \rightarrow C_{0}^{\prime}$ be the unique homeomorphism between $C_{0}$ and $C_{0}^{\prime}$.

Suppose for any $k=0,1, \ldots$, that $B_{k} \subseteq C_{k}, B_{k}^{\prime} \subseteq C_{k}^{\prime}$, and there is a homeomorphism

$$
f_{k}:\left(C_{k}, \text { fr } C_{k}\right) \rightarrow\left(C_{k}^{\prime}, \text { fr } C_{k}^{\prime}\right)
$$

where either $C_{k}=C_{k}^{\prime}=\varnothing$ or $C_{k} \subseteq M$ and $C_{k}^{\prime} \subseteq M^{\prime}$ are submanifolds that satisfy properties (1), (2) and (3). Suppose also that $Q_{1}, \ldots, Q_{m}$ are the complementary domains of $C_{k}$, that $Q_{1}^{\prime}, \ldots, Q_{m}^{\prime}$ are the complementary domains of $C_{k}^{\prime}$, and, for $i=1, \ldots, m$, that $h$ and $f$ induce isomorphisms $\Delta Q_{i} \rightarrow \Delta Q_{i}^{\prime}$.

By Lemma 1.3 there is a submanifold $D^{\prime}$ of $M^{\prime}$ such that

$C_{k}^{\prime} \cup B_{k+1}^{\prime} \subseteq$ int $D^{\prime}$,

$D^{\prime}$ satisfies properties (1), (2), (3) and (4), and

$Q_{i}^{\prime} \cap D^{\prime}$ is connected.

By Lemma 2.1 there is a submanifold $D$ of $M$ such that

$C_{k} \subseteq$ int $D$, 
$D$ satisfies properties (1), (2) and (3),

$f_{k}$ extends to a homeomorphism $g:(D$, fr $D) \rightarrow\left(D^{\prime}\right.$, fr $\left.D^{\prime}\right)$, and

$h$ and $g$ induce isomorphisms $\Delta R_{j} \rightarrow \Delta R_{j}^{\prime}$ between the complementary domains $R_{1}, \ldots, R_{n}$ of $D$ and the complementary domains $R_{1}^{\prime}, \ldots, R_{n}^{\prime}$ of $D^{\prime}$.

Now reversing the roles of $M$ and $M^{\prime}$ and putting $D$ in the place of $C_{k}^{\prime}$, we obtain from Lemma 1.3 a submanifold $C_{k+1}$ of $M$ with properties corresponding to those listed above for $D^{\prime}$. Then Lemma 2.1 gives a submanifold $C_{k+1}^{\prime}$ of $M^{\prime}$ and a homeomorphism

$$
f_{k+1}:\left(C_{k+1}, \text { fr } C_{k+1}\right) \rightarrow\left(C_{k+1}^{\prime}, \text { fr } C_{k+1}^{\prime}\right)
$$

that extends $g$ and gives the desired isomorphisms between diagrams of corresponding complementary domains.

Repeating this process inductively will yield exhausting sequences $C_{n}$ and $C_{n}^{\prime}, n=0,1, \ldots$, for $M$ and $M^{\prime}$ respectively and homeomorphisms $f_{n}$ : $C_{n} \rightarrow C_{n}^{\prime}$ with the property that $f_{n+1} \mid C_{n}=f_{n}$. Thus the union of these homeomorphisms will be a homeomorphism between $M$ and $M^{\prime}$.

3. Signed graphs. The construction of a 2-manifold with a given set of invariants involves some graph theory of finite 1-dimensional $\mathrm{CW}$ complexes. These are known as multigraphs and are often defined in terms of multisets. The following less common but equivalent definition is better suited to our application.

A graph $\Gamma$ consists of three finite sets and two functions:

$$
\varepsilon \stackrel{e}{\leftarrow} \stackrel{v}{\rightarrow} \mathscr{T}
$$

where $e$ is two-to-one and onto. An edge is an element of $\mathcal{E}$, a vertex is an element of $\mathcal{V}$, and $I \in \mathcal{G}$ is an incidence of the edge $e(I)$ with the vertex $v(I)$.

Notice that graphs can be added by means of the operation of disjoint union; the empty graph acts as the identity. A graph is connected if and only if it cannot be written as a nontrivial disjoint union. A component of a graph is a maximal connected subgraph.

A graph is even if and only if, for each vertex $V, v^{-1}(V)$ has an even number of elements. A signed graph is an even graph together with a specified subset of vertices, called signed vertices, and disjoint subsets $\mathrm{G}^{-}$and $\mathrm{g}^{+}$of incidences such that for each signed vertex $V, v^{-1}(V) \subseteq g^{-} \cup g^{+}$and the number of elements in $v^{-1}(V) \cap G^{-}$is equal to the number of elements in $v^{-1}(V) \cap \mathscr{G}^{+}$. Notice that an edge of a signed graph may have both incidences in $\mathcal{G}^{-}$or in $\mathcal{G}^{+}$, or it may even join a signed vertex to a vertex that is not signed.

A path in a signed graph is a nonempty sequence $I_{1}, \ldots, I_{2 r}$ of distinct 
incidences such that, for $k=1, \ldots, r, e\left(I_{2 k-1}\right)=e\left(I_{2 k}\right)$; for $k=1, \ldots, r-$ $1, v\left(I_{2 k}\right)=v\left(I_{2 k+1}\right)$ and if $v\left(I_{2 k}\right)$ is a signed vertex, then $I_{2 k} \in \mathcal{G}^{-}$if and only if $I_{2 k+1} \in g^{+}$. A circuit is a path $I_{1}, \ldots, I_{2 r}$ such that $v\left(I_{1}\right)=v\left(I_{2 r}\right)$ and if $v\left(I_{2 r}\right)$ is a signed vertex, then $I_{2 r} \in \mathcal{G}^{-}$if and only if $I_{1} \in \mathscr{G}^{+}$. A set of circuits of a graph is complete provided every incidence of the graph occurs in exactly one of the circuits. A circuit is Eulerian provided it forms a complete set for some component of the graph. An Eulerian system for a graph is a complete set of Eulerian circuits for the graph.

TheOREM 3.1. Suppose $\Sigma$ is a set of disjoint circuits of a signed graph. Then there is a complete set of circuits that contains $\Sigma$.

Proof. We first want to consider paths that are disjoint from the circuits of $\Sigma$. If $\Sigma$ is not a complete set of circuits, then the two incidences of some edge will form such a path. Suppose $I_{1}, \ldots, I_{2 r}$ is any such path. If $I_{1}, \ldots, I_{2 r}$ is not a circuit, then, since the graph is even, we can find an incidence $I_{2 r+1}$ that is distinct from $I_{1}, \ldots, I_{2 r}$ and the incidences of the circuits of $\Sigma$ and so that $v\left(I_{2 r}\right)=v\left(I_{2 r+1}\right)$. If $v\left(I_{2 r}\right)$ is a signed vertex, we can also require that $I_{2 r+1} \in G^{+}$if and only if $I_{2 r} \in 9^{-}$. Let $I_{2 r+2}$ be the other incidence of $e^{-1}\left(e\left(I_{2 r+1}\right)\right)$. Then the longer path $I_{1}, \ldots, I_{2 r}, I_{2 r+1}, I_{2 r+2}$ will still be disjoint from the circuits of $\Sigma$. This process will eventually produce a circuit disjoint from the circuits of $\Sigma$. The conclusion of the theorem now follows by induction on the number of incidences of the graph that are not in circuits of $\Sigma$.

We now want to combine circuits of a signed graph to form Eulerian circuits. In addition we want a certain compatibility between the orders given to the incidences by the original circuits and by the resulting Eulerian circuits. The following definitions make this idea precise.

Let $X$ be the disjoint union of a countable number of circles $X_{1}, X_{2}, \ldots$ A circular order on a finite set $F$ is an equivalence class [ $f]$ of injections $f$ : $F \rightarrow X$ where $f$ is equivalent to $g$ provided there is a homeomorphism $\mu$ : $X \rightarrow X$ with $g=\mu \circ f$. A circuit of a circular order $[f]$ is a set $f^{-1}\left(X_{i}\right)$. A circular order $[g]$ is stronger than a circular order $[f]$ if and only if

(i) for any circuit $F_{i}$ of $[f]$, the circular orders $\left[g \mid F_{i}\right]$ and $\left[f \mid F_{i}\right]$ are equivalent, and

(ii) for any two distinct circuits $F_{i}$ and $F_{j}$ of $[f], g\left(F_{j}\right)$ is contained in one of the components of $X-g\left(F_{i}\right)$.

Notice that if $[g]$ is stronger than $[f]$, then the circuits of $[g]$ are unions of circuits of $[f]$.

LEMMA 3.2. Suppose $[f],[g]$, and $[h]$ are circular orders on a finite set $F$. If $[h]$ is stronger than $[g]$ and $[g]$ is stronger than $[f]$, then $[h]$ is stronger than $[f]$. 
Proof. Let $F_{i}$ be a circuit of $[f]$ and let $G_{i}$ be the circuit of $[g]$ that contains $F_{i}$. There are homeomorphisms $\mu, \nu: X \rightarrow X$ such that $g \mid F_{i}=\mu \circ\left(f \mid F_{i}\right)$ and $h \mid G_{i}=\nu \circ\left(g \mid G_{i}\right)$. Thus $h \mid F_{i}=\nu \circ\left(g \mid F_{i}\right)=\nu \circ \mu \circ\left(f \mid F_{i}\right)$, so $h \mid F_{i}$ is equivalent to $f \mid F_{i}$. Suppose now that $F_{j}$ is a circuit of $[f]$ distinct from $F_{i}$. Let $G_{j}$ be the circuit of $[g]$ that contains $F_{j}$. Suppose that $G_{i}=G_{j}$. Since $g\left(F_{j}\right)$ is contained in one of the components of $X-g\left(F_{i}\right)$, we have that

$$
h\left(F_{j}\right)=\nu \circ\left(g \mid G_{i}\right)\left(F_{j}\right)=\nu\left(g\left(F_{j}\right)\right)
$$

is contained in one of the components of

$$
\nu\left(X-g\left(F_{i}\right)\right)=X-\nu\left(g\left(F_{i}\right)\right)=X-\nu \circ\left(g \mid G_{i}\right)\left(F_{i}\right)=X-h\left(F_{i}\right) .
$$

Suppose that $G_{i} \neq G_{j}$. Since $h\left(F_{i}\right) \subseteq h\left(G_{i}\right), h\left(F_{j}\right) \subseteq h\left(G_{j}\right)$, and $h\left(G_{j}\right)$ is contained in one of the components of $X-h\left(G_{i}\right)$, we have that $h\left(F_{j}\right)$ is contained in one of the components of $X-h\left(F_{i}\right)$.

A complete set $\Sigma$ of circuits for a signed graph determines a circular order [f] on the set of incidences as follows. Choose a distinct component $X_{j}$ of $X$ for each circuit $I_{1}, \ldots, I_{2 r}$ of $\Sigma$. Then define $f$ so that, for $k=1, \ldots, 2 r-$ $1, f\left(I_{k}\right)$ and $f\left(I_{k+1}\right)$ are adjacent on $X_{j}$.

TheOREM 3.3. Suppose $\Sigma$ is a complete set of circuits for a signed graph $\Gamma$. There is an Eulerian system for $\Gamma$ that determines a circular order on 9 stronger than the circular order determined by $\Sigma$.

Proof. If there is a circuit of $\Sigma$ that is not Eulerian, then there must be two circuits $I_{1}, \ldots, I_{2 r}$ and $J_{1}, \ldots, J_{2 s}$ of $\Sigma$ with a common vertex $v\left(I_{2 k}\right)=$ $v\left(J_{2 l}\right)$. If this is a signed vertex, then

$$
I_{1}, \ldots, I_{2 k}, J_{2 l+1}, \ldots, J_{2 s}, J_{1}, \ldots, J_{2 l}, I_{2 k+1}, \ldots, I_{2 r}
$$

is a circuit if $I_{2 k}$ and $J_{2 l}$ have the same sign, while

$$
I_{1}, \ldots, I_{2 k}, J_{2 l}, J_{2 l-1}, \ldots, J_{1}, J_{2 s}, \ldots, J_{2 l+1}, I_{2 k+1}, \ldots, I_{2 r}
$$

is a circuit if $I_{2 k}$ and $J_{2 l}$ have opposite signs. If this common vertex is not a signed vertex, then both of the above are circuits.

This process of combining two circuits of $\Sigma$ yields a complete set of circuits for $\Gamma$ that determines a stronger circular order on 9 . Repetition of this process reduces the number of circuits and eventually produces the desired Eulerian system.

4. Abstract diagrams. In this section we establish characteristic properties of 2-manifold diagrams. We then study diagrams abstractly, and for diagrams with the characteristic properties, we establish results that will be used in the construction of a 2-manifold with the given diagram.

Let $M$ be a 2-manifold and let $V$ be an open and closed subset of $\pi_{0}(M)$. Consider the components of $d M$ that have a single end corresponding to an end in $V$. We define $S(V)$ to be the subset of $\underline{\pi}_{0}(d M)$ consisting of all such 
ends. If $E$ is a subset of $\pi_{0}(d M)$, we define $S_{E}(V)$ to be the union of $S(V)$ and the ends of elements of $E$ that correspond to ends in $V$. Let $\mathscr{P}$ be an orientation of $\pi_{0}(d M)$. Consider the components of $d M$ that have two ends corresponding to ends in $V$, and with both ends in $\mathscr{P}$ or neither end in $\mathscr{P}$. We define $T(V)$ to be the subset of $\underline{\pi}_{0}(d M)$ consisting of all such ends.

THEOREM 4.1. Suppose $M$ is a 2-manifold and $\mathcal{P}$ is an orientation of $\underline{\pi}_{0}(d M)$. Suppose $V$ is an open and closed subset of $\pi_{0}(M)$. Then

(i) $S(V)$ is finite and has an even number of elements,

(ii) if $V \cap \mathcal{K}=\varnothing$, then $T(V)$ is finite and $(S(V) \cup T(V)) \cap \mathscr{P}$ has the same number of elements as $(S(V) \cup T(V)) \cap\left(\pi_{0}(d M)-\mathscr{P}\right)$, and

(iii) if $V \cap \mathcal{K}=\varnothing$, then for any finite subset $E$ of $\pi_{0}(d M)$, there is a partition $V=V_{1} \cup \cdots \cup V_{k}$ such that, for each $i=1, \ldots, k, V_{i}$ is open, $T\left(V_{i}\right)=\varnothing$, and $S_{E}\left(V_{i}\right)$ has zero or two elements.

Proof. We can use Lemmas 1.2 and 1.3 to obtain a submanifold $C$ of $M$ so that $V=Q_{1}^{*} \cup \cdots \cup Q_{k}^{*}$ where $Q_{1}, \ldots, Q_{k}$ are certain complementary domains of $C$ and so that $C$ satisfies properties (1), (2), (3) and (4) listed in $\S 1$. Let $Q=Q_{1} \cup \cdots \cup Q_{k}$.

Consider the set of ends in $\underline{\pi}_{0}(d M)$ that correspond to ends of components of $d Q$ that intersect $C$. Notice that this set is finite, has an even number of elements, and contains $S(V)$. Of these, the ends that are not in $S(V)$ are the pairs of ends of components of $d M$ that are contained in $Q \cup C$. It follows that $S(V)$ is finite and has an even number of elements.

Suppose now that $V \cap \mathscr{K}=\varnothing$. By property (2c), $Q$ is orientable; by property (3), each $Q_{i}$ has an orientation that agrees with $\mathscr{P}$. Thus of the ends of components of $d Q$ that intersect $C$, one half correspond to ends in $\mathscr{P}$ and the other half correspond to ends in $\pi_{0}(d M)-\mathscr{P}$. Notice that these corresponding ends in $\pi_{0}(d M)$ contain $S(\overline{V)} \cup T(V)$. Of these, the ends that are not in $S(V) \cup T(V)$ are the pairs of ends of components of $d M$ that are contained in $Q \cup C$ and have exactly one end in $\mathscr{P}$. Conclusion (ii) thus follows.

Finally suppose $V \cap \mathcal{C}=\varnothing$ and a finite subset $E$ of $\pi_{0}(d M)$ is given. We can assume $C$ was chosen so that it intersects each element of $E$. By property (2b), $Q$ is planar; by property (3), each $Q_{i}$ has an orientation that agrees with $\mathcal{P}$. For $i=1, \ldots, k$, letting $V_{i}=Q_{i}^{*}$ partitions $V$ into disjoint open sets. Notice that, for each $i=1, \ldots, k$, fr $Q_{i}$ is connected. Otherwise we could adjoin to $C$ a strip in some $Q_{i}$ that joins two components of $\operatorname{fr} Q_{i}$. By property (4), this strip does not separate $Q_{i}$. Since the resulting submanifold still satisfies property (4), this process could be repeated. This would contradict the fact that $Q_{i}$ is planar. Now by arguments similar to those used to establish (i) and (ii), we conclude that $S_{E}\left(V_{i}\right)$ has zero or two elements and $T\left(V_{i}\right)=\varnothing$. 
An abstract 2-manifold diagram consists of the following spaces and maps.

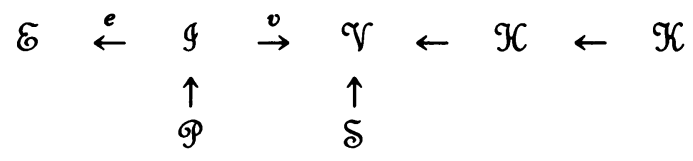

The spaces $\mathcal{E}$ and $\mathscr{G}$ are countable and discrete, $\mathcal{V}$ is a compact, totally disconnected, second countable Hausdorff space, and $\mathcal{K}, \mathcal{K}$, and $\mathcal{S}$ are closed subsets of $\mathcal{T}$. The unlabeled maps are inclusions, and $e$ is two-to-one and onto. If $I \in \mathcal{G}$, we use $I^{\prime}$ to denote the other element of $e^{-1}(e(I))$. We require that the analogue of Theorem 4.1 hold. Specifically if $V$ is any open and closed subset of $\mathscr{V}$, then

(i) $S(V)=\left\{I \in \mathscr{I} \mid v(I) \in V, v\left(I^{\prime}\right) \notin V\right\}$ is a finite set with an even number of elements,

(ii) if $V \cap \mathscr{K}=\varnothing$, then

$$
T(V)=\left\{I \in \mathscr{G} \mid v(I), v\left(I^{\prime}\right) \in V \text { and either } I, I^{\prime} \in \mathcal{P} \text { or } I, I^{\prime} \notin \mathscr{P}\right\}
$$

is a finite set and $(S(V) \cup T(V)) \cap \mathscr{P}$ has the same number of elements as $(S(V) \cup T(V)) \cap(\mathcal{G}-\mathcal{P})$, and

(iii) if $V \cap \mathcal{H}=\varnothing$, then for any finite subset $E$ of $\mathcal{E}$ there is a partition $V=V_{1} \cup \cdots \cup V_{k}$ such that, for each $i=1, \ldots, k, V_{i}$ is open, $T\left(V_{i}\right)=\varnothing$, and

$$
S_{E}\left(V_{i}\right)=S\left(V_{i}\right) \cup\left(e^{-1}(E) \cap v^{-1}\left(V_{i}\right)\right)
$$

has zero or two elements.

We next want to represent an abstract 2-manifold diagram as an inverse limit of signed graphs. For each $n=1,2, \ldots$, choose inductively a finite partition $\mathcal{V}_{n}$ of $\mathcal{V}$ and then a finite subset $\mathcal{E}_{n}$ of $\mathcal{E}$ that satisfy the following requirements. First, $\mathfrak{V}_{n}$ consists of open subsets of $\mathfrak{T}, \mathcal{V}_{n+1}$ refines $\mathfrak{V}_{n}$, and $\cup_{n=1}^{\infty} \mathcal{V}_{n}$ is a basis for the topology of $\mathfrak{V}$. Using property (iii) above, we also require that if $V \in \mathscr{V}_{n+1}$ and $V \cap \mathcal{H}=\varnothing$, then $T(V)=\varnothing$ and $S_{\mathscr{\varpi}_{n}}(V)$ has zero or two elements. The subsets $\mathcal{E}_{n}$ are finite, $\mathcal{E}_{n} \subseteq \mathcal{E}_{n+1}$, and $\cup_{n=1}^{\infty} \mathcal{E}_{n}=$ $\mathcal{E}$. Using property (i) above, we require for each $V \in \mathcal{V}_{n}$ that $e(S(V)) \subseteq \mathcal{E}_{n}$; using property (ii) above, we require for each $V \in \mathscr{V}_{n}$ with $V \cap \mathcal{K}=\varnothing$ that $e(T(V)) \subseteq \varepsilon_{n}$.

Let $g_{n}=e^{-1}\left(\mathcal{E}_{n}\right)$, let $e_{n}: \mathscr{G}_{n} \rightarrow \mathcal{E}_{n}$ be the restriction of $e$, and let $v_{n}$ : $\mathscr{G}_{n} \rightarrow \mathcal{V}_{n}$ be the restriction of $v$ composed with the projection from $\mathfrak{V}$ onto the quotient space $\mathscr{V}_{n}$. Let $\mathscr{G}_{n}^{+}=\mathscr{G}_{n} \cap \mathscr{P}$ and $\mathscr{G}_{n}^{-}=\mathscr{G}_{n}-\mathscr{P}$. Properties (i) and (ii) imply that

$$
\varepsilon_{n} \stackrel{e_{n}}{\leftarrow} g_{n} \stackrel{v_{n}}{\rightarrow} \mathscr{V}_{n}
$$

is a signed graph $\Gamma_{n}$ where the signed vertices are the elements of $\mathfrak{V}_{n}$ that are disjoint from $\mathcal{K}$. 
LEMMA 4.2. If the sequence $I_{1}, \ldots, I_{2 r}$ is a path in the signed graph $\Gamma_{n+1}$, then the subsequence $I_{j_{1}}, \ldots, I_{j_{k}}$ of elements of $G_{n}$ is a path in the signed graph $\Gamma_{n}$.

Proof. Let $I_{s}$ and $I_{t}$ be consecutive incidences in the subsequence. If $s$ is odd, then $I_{s}$ is an incidence of an edge in $\mathcal{E}_{n}$. Thus $t=s+1$ and $e_{n}\left(I_{s}\right)=$ $e_{n}\left(I_{t}\right)$. If $s$ is even, then notice for $i=s, \ldots, t$, that $v_{n+1}\left(I_{i}\right) \subseteq v_{n}\left(I_{s}\right)$. This is because $e\left(S\left(v_{n}\left(I_{s}\right)\right)\right) \subseteq \mathcal{E}_{n}$ and $\mathcal{V}_{n+1}$ is a refinement of $\mathfrak{V}_{n}$. In particular $v_{n}\left(I_{s}\right)=v_{n}\left(I_{t}\right)$. Furthermore if $v_{n}\left(I_{s}\right)$ is disjoint from $\mathcal{K}$ and is thus a signed vertex, then $I_{s}, I_{s+1}, \ldots, I_{t}$ are alternately in $\mathscr{G}_{n+1}^{-}$and $G_{n+1}^{+}$. This is because $e\left(T\left(v_{n}\left(I_{s}\right)\right)\right) \subseteq \mathcal{E}_{n}$ and for $i=s, \ldots, t$, each $v_{n+1}\left(I_{i}\right)$ is a signed vertex. It follows, since $t-s$ must be odd, that $I_{s} \in \mathscr{Y}_{n}^{-}$if and only if $I_{t} \in \mathscr{G}_{n}^{+}$.

The proof of Lemma 4.2 can easily be extended to prove the following lemma.

LEMMA 4.3. If $\sigma$ is a circuit of the signed graph $\Gamma_{n+1}$, then the subsequence of incidences of $\sigma$ that are elements of $G_{n}$ forms a circuit of the signed graph $\Gamma_{n}$. The vertices associated with $\sigma$ are contained in the vertices associated with the circuit of $\Gamma_{n}$.

It follows from Theorems 3.1 and 3.3 that for each $n=1,2, \ldots$, there exists an Eulerian system $\Sigma_{n}$ for the signed graph $\Gamma_{n}$. If $m<n$, then by Lemma 4.3, the restriction of the circuits of $\Sigma_{n}$ to the incidences in $G_{m}$ yields a complete set $\Sigma_{m}^{\prime}$ of circuits for $\Gamma_{m}$. The system $\Sigma_{m}$ is consistent with the system $\Sigma_{n}$ if and only if the circular order of $G_{m}$ determined by $\Sigma_{m}$ is stronger than that determined by $\Sigma_{m}^{\prime}$.

LEMMA 4.4. There is an Eulerian system $\Sigma_{n}$ for each of the signed graphs $\Gamma_{n}$ such that, for $n=1,2, \ldots, \Sigma_{n}$ is consistent with $\Sigma_{n+1}$.

Proof. We can apply Theorem 3.3 to the complete set of circuits that result from restricting to $G_{n}$ an Eulerian system for $\Gamma_{n+1}$. The resulting Eulerian system for $\Gamma_{n}$ is consistent with the system for $\Gamma_{n+1}$. These steps can be repeated to obtain Eulerian systems for $\Gamma_{n}, \Gamma_{n-1}, \ldots, \Gamma_{1}$ in turn such that each is consistent with the preceding system.

Notice that any graph has only a finite number of distinct Eulerian systems. Thus for arbitrarily large values of $n$, Eulerian systems for $\Gamma_{n}$ will yield the same Eulerian system $\Sigma_{1}$ for $\Gamma_{1}$ when the restriction process is applied. Similarly there must be an Eulerian system $\Sigma_{2}$ for $\Gamma_{2}$ that results from the restriction process applied to an infinite subsequence of the systems that produce $\Sigma_{1}$. The construction of $\Sigma_{1}$ guarantees that $\Sigma_{1}$ is consistent with $\Sigma_{2}$.

By taking subsequences and choosing Eulerian systems that occur an 
infinite number of times, we can inductively obtain the desired Eulerian systems.

Now let $\Sigma_{n}$ denote a fixed choice of Eulerian system for $\Gamma_{n}$ such that, for $n=1,2, \ldots, \Sigma_{n}$ is consistent with $\Sigma_{n+1}$. We will next choose simple closed curves in a 2-sphere $S^{2}$ to represent the circuits of the system $\Sigma_{n}$. Certain points on the curves will represent the incidences, and the arcs between them will represent the associated vertices and edges. The curves for $\Sigma_{n+1}$ will be nested within disks bounded by the curves for $\Sigma_{n}$ to reflect the way $\mathcal{E}_{n}$ is contained in $\mathcal{E}_{n+1}$ and the way $\mathfrak{V}_{n+1}$ refines $\mathfrak{V}_{n}$. The regions between the curves will then be used to construct a 2-manifold that realizes the abstract 2-manifold diagram. Figure 1 illustrates a typical stage in this construction.

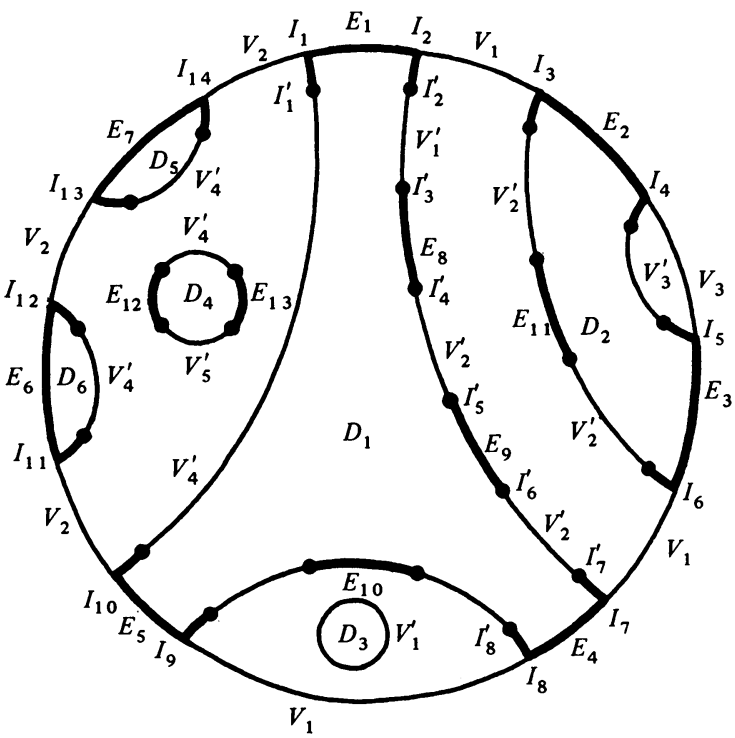

Figure 1. Labels on the disk $D$

For any $n=1,2, \ldots$, choose disjoint disks on $S^{2}$, one for each component of the graph $\Gamma_{n}$. The following construction is to be performed for each component $\Delta$ of $\Gamma_{n}$ and for each corresponding disk $D \subseteq S^{2}$.

On $\partial D$ label certain points $p_{i}$ with the incidences $I_{i}$ of $\Delta$. Let $f_{n}$ denote the labeling function, so that $f_{n}\left(p_{i}\right)=I_{i}$. Now $\partial D$ induces a circular order on the chosen points, and the Eulerian circuit in $\Sigma_{n}$ that traces out the component $\Delta$ determines a circular order on the incidences of $\Delta$. We require $f_{n}$ to induce an isomorphism between these circular orders.

We next label each component $A$ of $\partial D-f_{n}^{-1}\left(g_{n}\right)$ with an edge or a vertex of $\Delta$. If the endpoints of $\operatorname{cl} A$ are labeled with two incidences of an edge $E$, we let $f_{n}(A)=E$. Otherwise the endpoints are labeled with two incidences 
associated with the same vertex $V$, and we let $f_{n}(A)=V$. There are two special cases. If $\Delta$ has just two incidences, and thus one edge and one vertex, then $\partial D-f_{n}^{-1}\left(g_{n}\right)$ consists of two components with the same endpoints. We let $f_{n}$ label either one with the edge and the other with the vertex. If $\Delta$ is an isolated vertex, then let $f_{n}(\partial D)=V$.

Let $\Delta_{i}, i=1, \ldots, k$, be the components of $\Gamma_{n+1}$ that have a vertex contained in a vertex of $\Delta$. By Lemma 4.3 all the vertices of each $\Delta_{i}$, $i=1, \ldots, k$, are contained in the vertices of $\Delta$. Choose the disks $D_{i}$, $i=1, \ldots, k$ for the corresponding components $\Delta_{i}$ with $D_{i} \subseteq D$ and so that $D_{i} \cap \partial D$ equals the union of all the $\operatorname{arcs} \operatorname{cl}_{n}^{-1}(E)$ where $E$ is a common edge of $\Delta_{i}$ and $\Delta$. We choose $f_{n+1}$ so that for any edge $E$ common to $\Delta_{i}$ and $\Delta$, the points that $f_{n}$ labels with the incidences of $E$ are contained in $f_{n+1}^{-1}(E)$ and are adjacent to the endpoints of $f_{n+1}^{-1}(E)$ that $f_{n+1}$ labels with the respective incidences. Such embeddings are possible because $\Sigma_{n+1}$ is consistent with $\boldsymbol{\Sigma}_{n}$. We will later be more specific about the embeddings of disks $D_{i}$ that correspond to components $\Delta_{i}$ having no edges in common with $\Delta$.

LeMMA 4.5. $\partial D-\cup_{i=1}^{k} D_{i}=\partial D \cap f_{n}^{-1}\left(\mathscr{V}_{n}\right)$.

Proof. By the choice of $D_{i}$, we have that $\partial D-\cup_{i=1}^{k} D_{i} \subseteq \partial D \cap f_{n}^{-1}\left(\mathcal{V}_{n}\right)$. On the other hand, $\mathcal{E}_{n} \subseteq \mathcal{E}_{n+1}$ so every edge of $\Delta$ is also an edge of some $\Delta_{i}$.

For the remainder of this section, $X$ will denote a component of $D-$ $\bigcup_{i=1}^{k} D_{i}$.

LEMMA 4.6. There is a vertex $V$ in $\mathfrak{V}_{n}$ so that $X \cap \partial D \subseteq f_{n}^{-1}(V)$.

Proof. If $\Delta$ consists of an isolated vertex $V$, then $\partial D \subseteq f_{n}^{-1}(V)$ and the result follows immediately. Otherwise let $F$ be an arc of $\operatorname{fr} X$ that joins two components of $X \cap \partial D$. Notice that $F$ is also a frontier component of some $D_{i}$. Since no edge in $\mathcal{E}_{n+1}-\mathcal{E}_{n}$ has incidences corresponding to different vertices in $\mathcal{V}_{n}$, any component of $F-f_{n+1}^{-1}\left(\mathcal{G}_{n+1}\right)$ that is labeled with an edge will have its endpoints labeled with incidences that correspond to the same vertex in $\mathfrak{V}_{n}$. Also any component of $F-f_{n+1}^{-1}\left(G_{n+1}\right)$ that is labeled with a vertex in $\mathfrak{V}_{n+1}$ will have its endpoints labeled with incidences corresponding to that vertex; so that incidences will correspond to the same vertex in $\mathfrak{V}_{n}$. Let $p_{j} \in f_{n}^{-1}\left(g_{n}\right)$ denote an endpoint of $F$. One of the endpoints of the component of $\partial D_{i}-f_{n+1}^{-1}\left(G_{n+1}\right)$ that contains $p_{j}$ will be a point $p_{k}^{\prime} \in$ $f_{n+1}^{-1}\left(g_{n+1}\right)$ with $f_{n}\left(p_{j}\right)=f_{n+1}\left(p_{k}^{\prime}\right)$. In particular if the component of $X \cap \partial D$ containing $p_{j}$ is labeled with the vertex $V$, then

$$
v_{n+1}\left(f_{n+1}\left(p_{k}^{\prime}\right)\right) \subseteq v_{n}\left(f_{n}\left(p_{j}\right)\right)=V \text {. }
$$

It follows then that the components of $X \cap \partial D$ joined by $F$ are labeled with the same vertex of $\mathfrak{V}_{n}$.

Now $X$ is a punctured disk and exactly one of its boundary components 
intersects $\partial D$. This outer component of $\partial X$ consists of arcs of $X \cap \partial D$ alternating with arcs of $\operatorname{fr} X$ which span $D$. The above argument shows that all the components of $X \cap \partial D$ will be labeled with the same vertex of $\mathfrak{V}_{n}$.

We now extend the labeling function $f_{n}$ by letting $f_{n}(X)=V$ where $V$ is the vertex in $\mathfrak{V}_{n}$ such that $X \cap \partial D \subseteq f_{n}^{-1}(V)$.

For any $\Delta_{i}$ that has no edge in common with $\Delta$, all the vertices of $\Delta_{i}$ will be contained in a single vertex of $\Delta$. We require $D_{i}$ to be embedded in $D$ so that $\partial D_{i} \subseteq$ fr $X$ where $f_{n}(X)=V$. Notice that it is possible to choose this embedding so that any orientations of $X$ and $D_{i}$ agree.

We now come to the questions of orientation. An orientation of $D$ determines an initial point and a terminal point of each open arc of $\partial D-$ $f_{n}^{-1}\left(g_{n}\right)$. An orientation of $D$ agrees with $\mathcal{P}$ if and only if an incidence from $G_{n}^{+}$labels the initial point and an incidence from $G_{n}^{-}$labels the terminal point of each component of $\partial D-f_{n}^{-1}\left(g_{n}\right)$ that is labeled with an edge. On the other hand an orientation of the planar surface $X$ agrees with $\mathcal{P}$ if and only if an incidence from $\mathscr{G}_{n+1}^{-}$labels the initial point and an incidence from $\mathscr{G}_{n+1}^{+}$labels the terminal point of each component of $\partial X-f_{n+1}^{-1}\left(q_{n+1}\right)$ that is labeled with an edge or contains an arc of $X \cap \partial D$.

Lemma 4.7. Suppose $f_{n}(X) \cap \mathscr{K}=\varnothing$. Then there is an orientation of $X$ that agrees with $\mathscr{P}$.

Proof. Notice that the components of $\partial X-f_{n+1}^{-1}\left(g_{n+1}\right)$ alternate between being labeled with a vertex in $\mathfrak{V}_{n+1}$ and either being labeled with an edge in $\mathcal{E}_{n+1}$ or containing an arc of $X \cap \partial D$. Thus we only need to show that the points of $f_{n+1}^{-1}\left(G_{n+1}\right) \cap \partial X$ are labeled with incidences that are alternately in $\mathscr{G}_{n+1}^{-}$and $G_{n+1}^{+}$. Since there is only one component of $\partial X$ that contains arcs of $X \cap \partial D$, we can orient $X$ to agree with $\mathscr{P}$ along this outer boundary component and then choose the embeddings of any of the disks $D_{i}$ so that this orientation of $X$ agrees with $\mathscr{P}$ along $X \cap D_{i}$.

Recall from the proof of Lemma 4.6 that the vertices in $\mathcal{V}_{n+1}$ that label the components of $\partial X-f_{n+1}^{-1}\left(g_{n+1}\right)$ are all contained in the single vertex $f_{n}(X)$ in $\mathcal{V}_{n}$. Since all these vertices are thus disjoint from $\mathcal{K}$, they are also signed vertices of $\Gamma_{n+1}$. Therefore a component of $\partial X-f_{n+1}^{-1}\left(G_{n+1}\right)$ labeled by a vertex in $\mathcal{T}_{n+1}$ will have one of its ends labeled by an incidence in $\mathscr{Y}_{n+1}^{-}$and the other by an incidence in $\mathscr{G}_{n+1}^{+}$. Since $e\left(T\left(f_{n}(X)\right)\right) \subseteq \mathcal{E}_{n}$, it follows that each edge in $\mathcal{E}_{n+1}$ that labels a component of $\partial X-f_{n+1}^{-1}\left(G_{n+1}\right)$ will have one of its incidences in $G_{n+1}^{-}$and the other in $G_{n+1}^{+}$. Finally, the endpoints of any components of $\partial X-f_{n+1}^{-1}\left(g_{n+1}\right)$ that contains an arc of $X \cap \partial D$ will be labeled with the same incidences that label the respective endpoints of $X \cap \partial D$. But the interior of this arc of $X \cap \partial D$ is labeled with a signed 
vertex. Hence one of the incidences involved is in $G_{n+1}^{-}$and the other is in $g_{n+1}^{+}$.

Lemma 4.8. Suppose $f_{n}(X) \cap \mathscr{K}=\varnothing$. Suppose $X^{\prime}$ is a component of the complement in $D_{i}$ of the disks corresponding to the components of $\Gamma_{n+2}$. If $X^{\prime} \cap \operatorname{fr} X \neq \varnothing$, then an orientation of $X$ that agrees with $\mathcal{P}$ will induce an orientation on $X^{\prime}$ that agrees with $\mathcal{P}$.

Proof. Since $f_{n+1}\left(X^{\prime}\right) \subseteq f_{n}(X)$ and thus $f_{n+1}\left(X^{\prime}\right) \cap \mathscr{K}=\varnothing$, Lemma 4.7 implies there is an orientation of $X^{\prime}$ that agrees with $\mathscr{P}$. Since a component of $X^{\prime} \cap \operatorname{fr} X$ is a component of $X^{\prime} \cap \partial D_{i}$ as well as the closure of a component of $\partial X-f_{n+1}^{-1}\left(g_{n+1}\right)$ that is labeled with a vertex, it follows that the orientations of $X$ and $X^{\prime}$ that agree with $\mathscr{P}$ are compatible.

LEMMA 4.9. Suppose $V_{1}, \ldots, V_{m}$ are the vertices of $\Delta$. Suppose $\left(V_{1}\right.$ $\left.\cup \cdots \cup V_{m}\right) \cap \mathcal{K}=\varnothing$ and $T\left(V_{1} \cup \cdots \cup V_{m}\right)=\varnothing$. Then there is an orientation of $D$ that agrees with $\mathscr{P}$. This orientation induces orientations on each component $X$ of $D-\cup{ }_{i=1}^{k} D_{i}$ and on the disks $D_{i}$ that agree with $\mathscr{P}$.

Proof. The hypotheses of this lemma imply that each vertex of $\Delta$ is a signed vertex and that each edge of $\Delta$ has one incidence in $\mathscr{G}_{n}^{-}$and one in $\mathscr{G}_{n}^{+}$. Thus since the points of $\partial D \cap f_{n}^{-1}\left(G_{n}\right)$ are alternately labeled with incidences from $\mathscr{G}_{n}^{-}$and $\mathscr{G}_{n}^{+}, D$ has an orientation that agrees with $\mathscr{P}$.

Unless $\Delta$ consists of an isolated vertex, the components of $X \cap \partial D$ will be arcs whose interiors are labeled with the vertices of $\Delta$. Thus the orientation of $X$ that agrees with $\mathscr{P}$ is the same as the orientation induced from $D$.

The fact that the vertices of $\Delta_{i}$ are contained in the vertices of $\Delta$ and are thus disjoint from $\mathscr{K}$ implies that $D_{i}$ has an orientation that agrees with $\mathscr{P}$. Since $D_{i}$ will intersect some component $X$ of $D-\cup_{i=1}^{k} D_{i}$ in a common frontier component, it follows that this orientation of $D_{i}$ is also the same as the orientation induced from $D$.

Notice that if the hypotheses of Lemma 4.9 are satisfied by a component of $\Gamma_{1}$, then the corresponding disk can be embedded in $S^{2}$ so that the orientation induced by an orientation of $S^{2}$ will agree with $\mathscr{P}$. In particular, this will be the case for all the components of $\Gamma_{1}$ if $\mathcal{K}=\varnothing$ and $T(\mathscr{V})=\varnothing$.

Now whenever the hypotheses of Lemmas 4.7 or 4.9 are satisfied, we choose orientations of the respective submanifolds of $S^{2}$ that agree with $\mathcal{P}$. Lemmas $4.7,4.8$, and 4.9 imply that these orientations will be compatible on the union of all such submanifolds.

5. Construction of 2-manifolds. In this section we construct a 2-manifold with a given set of invariants. We begin by constructing a 2-manifold from a particular representation of an abstract diagram as an inverse limit of signed graphs. We then adjust this 2 -manifold by taking connected sums with tori, 
projective planes, and disks to obtain all the 2-manifolds with the given diagram.

THEOREM 5.1. Suppose

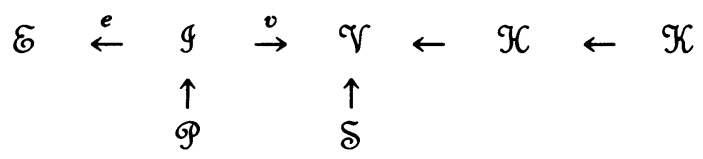

is an abstract 2-manifold diagram. Then there is a 2-manifold $M$ such that the diagram of $M$ is isomorphic to this abstract 2-manifold diagram. Furthermore

(i) if $\mathcal{H}=\varnothing$, then $M$ can be of any finite genus,

(ii) if $\mathcal{K}=\varnothing$, then $M$ can be of even or odd nonorientability type, and if $T(\mathfrak{V})=\varnothing$ also, then $M$ can be orientable, and

(iii) if $\mathcal{S}=\varnothing$, then $M$ can have any finite number of compact boundary components.

Proof. As defined in the previous section, for each $n=1,2, \ldots$, let $\Sigma_{n}$ be an Eulerian system for the signed graph $\Gamma_{n}$ and let $f_{n}$ be the labeling function defined on the various portions of the disks of $S^{2}$ that correspond to the components of $\Gamma_{n}$.

For each vertex $V \in \mathfrak{V}_{n}$, we want to join together the components of $f_{n}^{-1}(V)$ with tubes. That is, we remove the interiors of appropriate pairs of disks from $f_{n}^{-1}(V)$ and attach annuli along the resulting boundary components to form a connected, planar 2-manifold. This submanifold of the modified 2-sphere is still to be labeled with the vertex $V$. If $V \cap \mathcal{K}=\varnothing$, then we can attach the tubes so that there is an orientation of $f_{n}^{-1}(V)$ compatible with the orientations of the original components.

Let $C_{1}$ be the complement in $S^{2}$ of the interiors of the disks that correspond to components of $\Gamma_{1}$. For each $n=1,2, \ldots$, we inductively define $C_{n+1}$ to be the union of $C_{n}$ and $f_{n}^{-1}(V)$ for all vertices $V$ in $\mathfrak{V}_{n}$. The inclusion of $C_{n}$ into $C_{n+1}$ defines a direct system; let $M$ be the direct limit of this system. For $n=1,2, \ldots$, we can identify $C_{n}$ with a subset of $M$. Then $C_{1}$, $C_{2}, \ldots$ forms an exhausting sequence for the 2-manifold $M$.

For $n=1,2, \ldots$, the labeling function $f_{n}$ gives a bijection between $\pi_{0}\left(C_{n+1}-\right.$ int $\left.C_{n}\right)$ and $\mathfrak{V}_{n}$. Notice that for $V \in \mathfrak{V}_{n}$ and $V^{\prime} \in \mathfrak{V}_{n+1}$, we have $\operatorname{cl}\left(f_{n}^{-1}(V)\right) \cap \operatorname{cl}\left(f_{n+1}^{-1}\left(V^{\prime}\right)\right) \neq \varnothing$ if and only if $V^{\prime} \subseteq V$. Thus there is bijection between $\pi_{0}\left(C_{n+1}-\right.$ int $\left.C_{n}\right)$ and $\pi_{0}\left(M-\right.$ int $\left.C_{n}\right)$ induced by inclusion. The compositions of these bijections give the following isomorphism of the inverse systems, where the horizontal arrows indicate maps induced by inclusion.

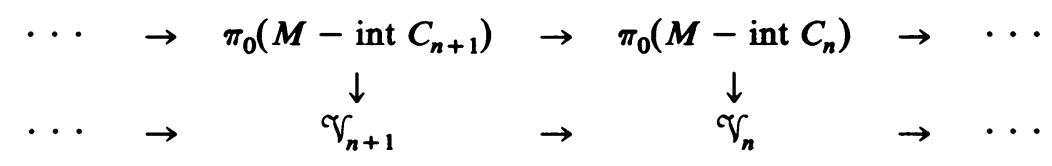


This yields a homeomorphism $f: \underline{\pi}_{0}(M) \rightarrow \mathcal{V}$ between the inverse limits of the respective systems.

Since $C_{1}, C_{2}, \ldots$, forms an exhausting sequence for $M, C_{1} \cap d M, C_{2} \cap$ $d M, \ldots$, forms an exhausting sequence for $d M$. Now the components of $\operatorname{int}\left(C_{n} \cap d M\right)$ are labeled with the edges in $\mathcal{E}_{n}$, and the labeling functions induce the following isomorphism of direct systems, where the horizontal arrows indicate maps induced by inclusion.

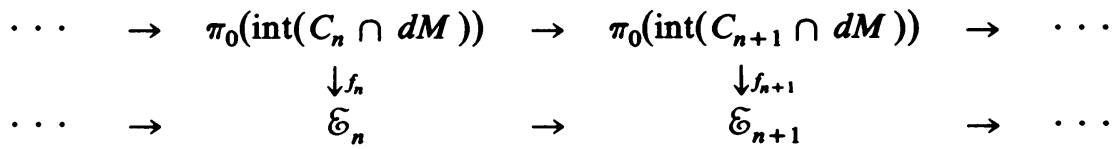

This yields a homeomorphism $f: \pi_{0}(d M) \rightarrow \mathcal{E}$ between the direct limits of the respective systems.

We can map endpoints of each component of $C_{n} \cap d M$ to the adjacent endpoints of the component of $C_{n+1} \cap d M$ that contains it. Now the endpoints of the components of $C_{n} \cap d M$ are labeled with the incidences in $G_{n}$, and the labeling functions induce the following isomorphism of direct systems.

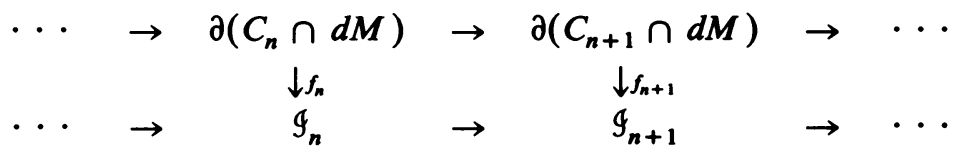

This yields a homeomorphism $f: \underline{\pi}_{0}(d M) \rightarrow G$ between the direct limits of the respective systems.

Any point of $\pi_{0}(d M)$ can be represented for some $n$ by an endpoint of a component of $\bar{C}_{n} \cap d M$. The element of $g_{n}$ that labels this point is an incidence of the edge that labels the interior of this component of $C_{n} \cap d M$. Thus the following diagram commutes.

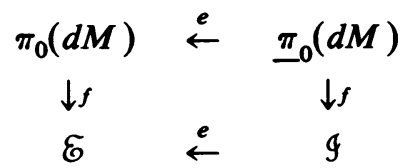

Again a point $x \in \pi_{0}(d M)$ can be represented by an endpoint $p$ of some component $A$ of $C_{n} \cap \overline{d M}$. For each $i=n, n+1, \ldots$, there is a component of $C_{i} \cap d M$ that contains $A$. The endpoint $p_{i}$ of this component that is adjacent to $p$ will also represent $x$. The points $p_{n}, p_{n+1}, \ldots$, form a monotone sequence on a proper ray of $d M$ that represents the end $v(x) \in \pi_{0}(M)$. Now for $i=n, n+1, \ldots, f_{i}\left(p_{i}\right)$ is an incidence of the vertex in $\overline{\mathcal{V}}_{i}$ that labels the component of $C_{i+1}-$ int $C_{i}$ containing $p_{i}$. Thus the sequence of components of $M$ - int $C_{i}$ that defines $v(x)$ corresponds to the sequence of vertices in $\mathfrak{V}_{i}$ that defines $v(f(x))$. Therefore $f(v(x))=v(f(x))$ and hence the 
following diagram commutes

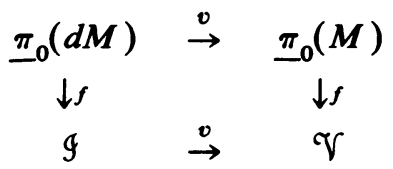

Consider $x \in \pi_{0}(M)$ such that $f(x) \notin \mathcal{H}$. For some $n$, we will have $f(x) \in V \in \mathcal{V}_{n}$ and $V \cap \mathcal{H}=\varnothing$. Recall that $v_{n}^{-1}(V)$ has zero or two points. Thus in the boundary of the disks in $S^{2}$ corresponding to the components of $\Gamma_{n}$, there is only one component of the complement of $f_{n}^{-1}\left(g_{n}\right)$ that is labeled with $V$. Since $f_{n}^{-1}(V) \cap C_{n}$ consists of the closure of such components, it follows from Lemma 4.6 that $f_{n}^{-1}(V) \cap C_{n}$ is connected. Similarly for any $i=n, n+1, \ldots$, and for any vertex $V^{\prime} \in \mathcal{V}_{i}$ with $V^{\prime} \subseteq V$, we will have that $f_{i}^{-1}\left(V^{\prime}\right) \cap C_{i}$ is connected. Since the component of $M-$ int $C_{n}$ that contains $f_{n}^{-1}(V)$ is the union of these planar submanifolds, this component will be planar. Now $f^{-1}(V)$ is the set of ends of $M$ determined by this component of $M-$ int $C_{n}$. Therefore $x$ is a planar end of $M$. We conclude that $f^{-1}(\mathcal{H})$ contains the nonplanar ends of $M$.

Consider $x \in \pi_{0}(M)$ such that $f(x) \notin \mathscr{K}$. For some $n$, we will have $f(x) \in$ $V \in \mathcal{V}_{n}$ and $V \bar{\cap} \mathscr{K}=\varnothing$. For any $i=n, n+1, \ldots$ and for any vertex $V^{\prime} \in \mathfrak{V}_{i}$ with $V^{\prime} \subseteq V$, we have that $f_{i}^{-1}\left(V^{\prime}\right)$ is orientable. This follows from Lemma 4.7 and the way the tubes were attached to form $f_{i}^{-1}\left(V^{\prime}\right)$. As remarked in the last paragraph of the previous section, orientations can be chosen to be compatible on the union of these submanifolds. Thus the component of $M-$ int $C_{n}$ that contains $f_{n}^{-1}(V)$ is orientable. Therefore $x$ is an orientable end of $M$. We conclude that $f^{-1}(\mathscr{K})$ contains the nonorientable ends of $M$.

Notice that at this stage in the construction $M$ has no compact boundary components. In particular no ends of $M$ are limits of compact boundary components.

Choose sequences of components of $C_{n+1}-$ int $C_{n}, n=1,2, \ldots$, that define any planar ends of $M$ that are contained in $f^{-1}(\mathcal{H})$, any orientable ends of $M$ that are contained in $f^{-1}(\mathcal{K})$, and any ends of $M$ that are contained in $f^{-1}(\delta)$. Modify each such component by taking the connected sum of it with a torus, a projective plane, or a disk so that for the resulting 2-manifold, $f^{-1}(\mathcal{K}), f^{-1}(\mathcal{K})$, and $f^{-1}(\mathcal{S})$ will be the set of nonplanar ends, the set of nonorientable ends, and the set of ends that are limits of compact boundary components, respectively.

Now let $x$ denote an orientable end in $\pi_{0}(M)$. Since $f(x) \notin \mathscr{K}$, for some $n$ we will have $f(x) \in V \in \mathcal{V}_{n}$ with $V \cap \overline{\mathcal{K}}=\varnothing$. As remarked in the last paragraph of the previous section, it follows from Lemmas 4.7 and 4.8 that the component of $M-$ int $C_{n}$ that contains $f_{n}^{-1}(V)$ will be oriented so that for 
any $i=n, n+1, \ldots$ and for any vertex $V^{\prime} \in \mathscr{V}_{i}$ with $V^{\prime} \subseteq V$, the orientations induced on $f_{i}^{-1}\left(V^{\prime}\right)$ will agree with $\mathscr{P}$. In particular, for any portion of $\partial\left(f_{i}^{-1}\left(V^{\prime}\right)\right)$ that is labeled with an edge, the points of $f_{i}^{-1}\left(G_{i}^{-}\right) \cap \partial\left(f_{i}^{-1}\left(V^{\prime}\right)\right)$ will be the initial points while the points of $f_{i}^{-1}\left(g_{i}^{+}\right) \cap \partial\left(f_{i}^{-1}\left(V^{\prime}\right)\right)$ will be the terminal points. It follows that the orientation on this component of $M-$ int $C_{n}$ agrees with $f^{-1}(\mathscr{P})$. Notice that at this stage in the construction, if $\mathcal{K}=\varnothing$ and $T(\mathscr{V})=\varnothing$, then $M$ is orientable. Thus by the remarks at the end of the previous section, the orientation of $M$ agrees with $f^{-1}(\mathcal{P})$. If $T(\mathscr{V}) \neq \varnothing$, it is then necessary to modify $M$ by taking the connected sum of it with a projective plane to make it nonorientable. In any case we now conclude that $f^{-1}(\mathscr{P})$ is an orientation of $\pi_{0}(d M)$.

If $\mathcal{H}=\varnothing$, then $M$ is planar. By taking the connected sum of $M$ with tori, we can modify $M$ to have any finite genus.

If $\mathscr{K}=\varnothing$, then we can similarly take the connected sum of $M$ with projective planes and modify $M$ to have either even or odd nonorientability type.

If $\mathcal{S}=\varnothing$, then we can take the connected sum of $M$ with disks to obtain any finite number of compact boundary components.

\section{REFERENCES}

1. Magarida Barros, Classification of non-compact surfaces whose boundary is the union of Jordan curves, An. Fac. Ci. Univ. Porto. 57 (1974), 33-41.

2. E. M. Brown and T. W. Tucker, On proper homotopy theory for noncompact 3-manifolds, Trans. Amer. Math. Soc. 188 (1974), 105-126.

3. Martin E. Goldman, An algebraic classification of noncompact 2-manifolds, Trans. Amer. Math. Soc. 156 (1971), 241-258.

4. B. Kerékjärtó, Vorlesungen über Topologie, Vol. I, Springer, Berlin, 1923.

5. Ian Richards, On the classification of noncompact surfaces, Trans. Amer. Math. Soc. 106 (1963), 259-269.

6. Richard J. Tondra, Homeotopy groups of surfaces whose boundary is the union of 1-spheres, Fund. Math. (to appear).

Department of Mathematics, Dartmouth College, Hanover, New Hampshire 03755 (Current address of E. M. Brown)

Current address (Robert Messer): Department of Mathematics, Vanderbilt University, Nashville, Tennessee 37235 